\title{
Atlantic Forest succession over calcareous soil, Parque Estadual Turístico do Alto Ribeira - PETAR, SP $^{1}$
}

\author{
MARCOS PEREIRA MARINHO AIDAR ${ }^{2,6}$, JOÃO RUFFIN LEME DE GODOY ${ }^{3}$, \\ JANINE BERGMANN $^{4}$ and CARLOS ALFREDO JOLY ${ }^{5}$
}

(received: February 14, 2001; accepted: September 12, 2001)

\begin{abstract}
Atlantic Forest succession over calcareous soil, Parque Estadual Turístico do Alto Ribeira - PETAR, SP). The forest succession after abandonment of slash-and-burn agriculture over calcareous soil in Brazilian Atlantic Forest was assessed. This is one of the world's most threatened Biome, with only $8 \%$ remaining. The study area is located over calcareous soil inside the Alto Ribeira Touristic State Park (PETAR), southeast Brazil. The phytossociological survey showed a successional pattern dominated by species of Leguminosae, especially Piptadenia gonoacantha (Mart.) J.F. Macbr. This species occurs in calcareous soils as a substitute of Tibouchina pulchra (Cham.) Cogn. (Melastomataceae) that is the most usual dominant tree species in early succession over acidic soil, which is the most common situation in this Biome. These results are important for a better understanding of Neotropical forest biodiversity and characterize a unique genetic bank in this highly endangered Biome. They are also decisive to support actions regarding rehabilitation of degraded lands and a potential tool for Neotropical forest sustainable management, both inside and around the conservation unit.
\end{abstract}

RESUMO - (Sucessão de Mata Atlântica sobre solos calcários, Parque Estadual Turístico do Alto Ribeira - PETAR, SP). Foram levantadas as características da sucessão florestal após abandono de campo cultivado em agricultura de subsistência sobre solos calcários na Mata Atlântica brasileira. Este é um dos biomas mais ameaçados do mundo, que apresenta apenas 8\% de remanescentes. A área de estudo está localizada sobre solo calcário no Parque Estadual Turístico do Alto Ribeira (PETAR), sudeste do Brasil. O levantamento fitossociológico indicou padrão de sucessão dominado por Leguminosae, especialmente Piptadenia gonoacantha (Mart.) J.F. Macbr. Esta espécie ocorre sobre solo calcário, substituindo a espécie arbórea dominante em início de sucessão sobre solos ácidos, Tibouchina pulchra (Cham.) Cogn. (Melastomataceae), sendo que esta é a situação mais comum neste Bioma. Estes resultados são importantes para um melhor conhecimento da biodiversidade da floresta neotropical e caracteriza um banco genético único neste bioma altamente ameaçado. São também decisivos no suporte de ações de reabilitação de áreas degradadas e um instrumento potencial para manejo auto-sustentado da floresta neotropical, tanto dentro dos limites da unidade de conservação, quanto em suas áreas de entorno.

Key words - Secondary succession, Brazilian Atlantic Forest, tropical calcareous soil, Piptadenia gonoacantha, slash-andburn agriculture

\section{Introduction}

The Brazilian Atlantic Forest is considered one of the three most threatened ecosystems on Earth. Once covering more than a million squared kilometers, today the forest is reduced to less than $8 \%$ of the original cover (SOS Mata Atlântica 1998). It is considered by The Conservation International as one

1. Part of the Doctoral Thesis of M.P.M. Aidar.

2. Instituto de Botânica, Seção de Fisiologia e Bioquímica de Plantas, Caixa Postal 4005, 01061-970 São Paulo, SP, Brasil.

3. Universidade de São Paulo, Departamento de Ecologia, Caixa Postal 11461, 05422-970 São Paulo, SP, Brasil.

4. Centro Universitário FIEO, Ciências Biológicas, Av. Franz Voegeli, 300, 06020-190 Osasco, SP, Brasil.

5. Universidade Estadual de Campinas, Departamento de Botânica, Caixa Postal 6109, 13083-970 Campinas, SP, Brasil.

6. Corresponding author: maidar@uol.com.br of world's biodiversity hot spots (Myers et al. 2000). São Paulo State, SE Brazil, had more than $82 \%$ of its area covered by forest. Today, the remnants are around $7 \%$ of its original distribution (SOS Mata Atlântica 1998), occurring mainly in the mountainous region near the shore.

The Atlantic Forest is a complex of ecosystems that belongs to the Atlantic Dominion which includes a Dense Ombrophylous Forest (IBGE 1992, Joly et al. 1999 ) on slopes and mountain tops, especially in SE Brazil. This physiognomy is characterised as evergreen "hygrophyllous forest" (Torres et al. 1997), with high diversity and endemism indexes (Mori et al. 1981), and is considered as the richest forest for Orchidaceae and the most ancient diversity center for South America (Brieger 1969). In the Dense Ombrophylous Forest from southern and southeastern Brazil, the most common angiosperm families in terms of number of species (considering low and high altitude areas, below and above $700 \mathrm{~m}$ 
high, respectively) are: Myrtaceae, Leguminosae, Rubiaceae, Melastomataceae, Lauraceae, Sapotaceae, Euphorbiaceae, Moraceae and Annonaceae (Oliveira Filho \& Fontes 2000). Studies carried out in secondary forests over acidic and oligotrophic soils in this type of forest indicated the species Tibouchina pulchra (Cham.) Cogn. (Melastomataceae), an endemic genus (Veloso 1982), as one of the most important trees, if not the most, in these communities (Loefgren 1898, Leitão Filho et al. 1993, Tabarelli et al. 1993, 1994, 1999, Torezan 1995).

The gap-phase dynamics is the fundamental basis for studying the forest growth cycle and it is the beginning of the regenerative cycle. Disturbance created by gaps, that alter environmental conditions, triggers a construction phase characterised by species colonisation and growth following a continuum of ecophysiological response that induce cicatrization (Brokaw 1985). The final stage is the mature phase, where a new gap may restart the growth cycle (Whitmore 1984). The essential characteristic of shifting cultivation ("roça") is that slash-and-burn clearings are small (usually $<1 \mathrm{ha}$ ), and seed dispersal distances are short. During recovery phase, ecosystem function does not appear to be severely disrupted, and in this way, the abandoned "roças" are similar in behaving like big gaps (Uhl et al. 1990). Surveys of long-term succession in the Amazon produced a model on how tropical forest develops on abandoned farm sites, which could help in the design of ecologically sustainable uses of rain forest, e.g. through a natural succession-mimic aproach (Subler \& Uhl 1990), that prevent nutrient losses, protecting fragile soils while conserving the forest biological diversity (Gomez-Pompa \& Burley 1991).

Although difficult to be defined, secondary forests are estimated in a worldwide basis to cover between 530 (FAO 1996) to 600 million ha. (Brown \& Lugo 1990), roughly $33 \%$ of total tropical forest (7\% in Latin America). Based in this fact, Chazdon (1998) suggests that tropical secondary forests are now being recognised for their value in the conservation of biological diversity. Emrich et al. (2000) affirm that secondary forests are an inexpensive and siteappropriate form of reforestation and have in principle a high regenerative potential. They can fulfil a whole range of functions that can benefit humanity, including forestry uses (fuelwood, timber, non-timber forest products, tourism), agricultural uses (agroforestry systems) and environmental protection functions (water, soil and climate protection including $\mathrm{CO}_{2}$ sink functions and the conservation of biodiversity). With respect to carbon dioxide sequestration, a very important global issue nowadays, secondary forests are likely to mitigate global change due to the facts that plants are at early stages of development, showing higher $\mathrm{CO}_{2}$ assimilation and growth rates and that tree species are suitable for wood processing at older stages.

In the present work we performed the characterization of the species composition occurring in the main phases that drive the forest succession after slash-and-burn agriculture over calcareous soil through a phytosociological inventory. We observed a new pattern of Atlantic Forest succession related to the occurrence of calcareous soils, where legumes (especially Piptadenia gonoacantha) are dominant. This information will contribute to the knowledge about Atlantic Forest biodiversity and regeneration, and could help to develop a forest recovery model to support regional rehabilitation and management actions.

\section{Material and methods}

Study site - The study area is located in São Paulo State, southeastern of Brazil, at the Ribeira de Iguape Watershed, inside of the Parque Estadual Turístico do Alto Ribeira PETAR (2431'43" S; 4841'09" W), approximately 380 $\mathrm{km}$ southwest from São Paulo City, the biggest metropolitan area in South America (figure 1). This protected area (35712 ha) together with other state parks comprise more than 270000 ha of continuous Atlantic Forest in Conservation Units. This area was inscribed as Natural Site on the World Heritage List (www.unesco.org/whc/sites/ 893.htm). Regional climate is tropical hyperhumid without a dry period with mean annual precipitation around $1800 \mathrm{~mm}$, evenly distributed throughout the year $(34 \%$ summer and $17 \%$ winter of total annual precipitation) and mean annual temperature between $17-19^{\circ} \mathrm{C}\left(\max .23^{\circ} \mathrm{C}\right.$; min. $14^{\circ} \mathrm{C}$ ) (Gutjahr 1993). Geologically it has a preCambrian basement with many metamorphic degrees, mainly metasediments silicic-argillaceous and epimetamorphic calcareous. The relief is very mountainous with karstic plateaus and intrusive granites. The study site is located at 500-600 m above sea level over calcareous substrate, which comprises around $40 \%$ of the park area (figure 1), classified as Cambisols associated with Chernozems (Camargo et al. 1986, FAO-ISRIC-ISSS 1998). When compared with an acidic soil originated from a silicic-argillaceous substrate close to the study area (Torezan 1995), the eutrophic calcareous soil showed higher $\mathrm{pH}(\mathrm{pH}=6)$, saturation of bases $(\mathrm{V} \%=79)$, organic matter $(\mathrm{OM} \%=5)$ and calcium content $(9.5 \mathrm{mEq} / 100 \mathrm{ml})$, and much less exchangeable acidity $(2.6 \mathrm{mEq} / 100 \mathrm{ml})$. 

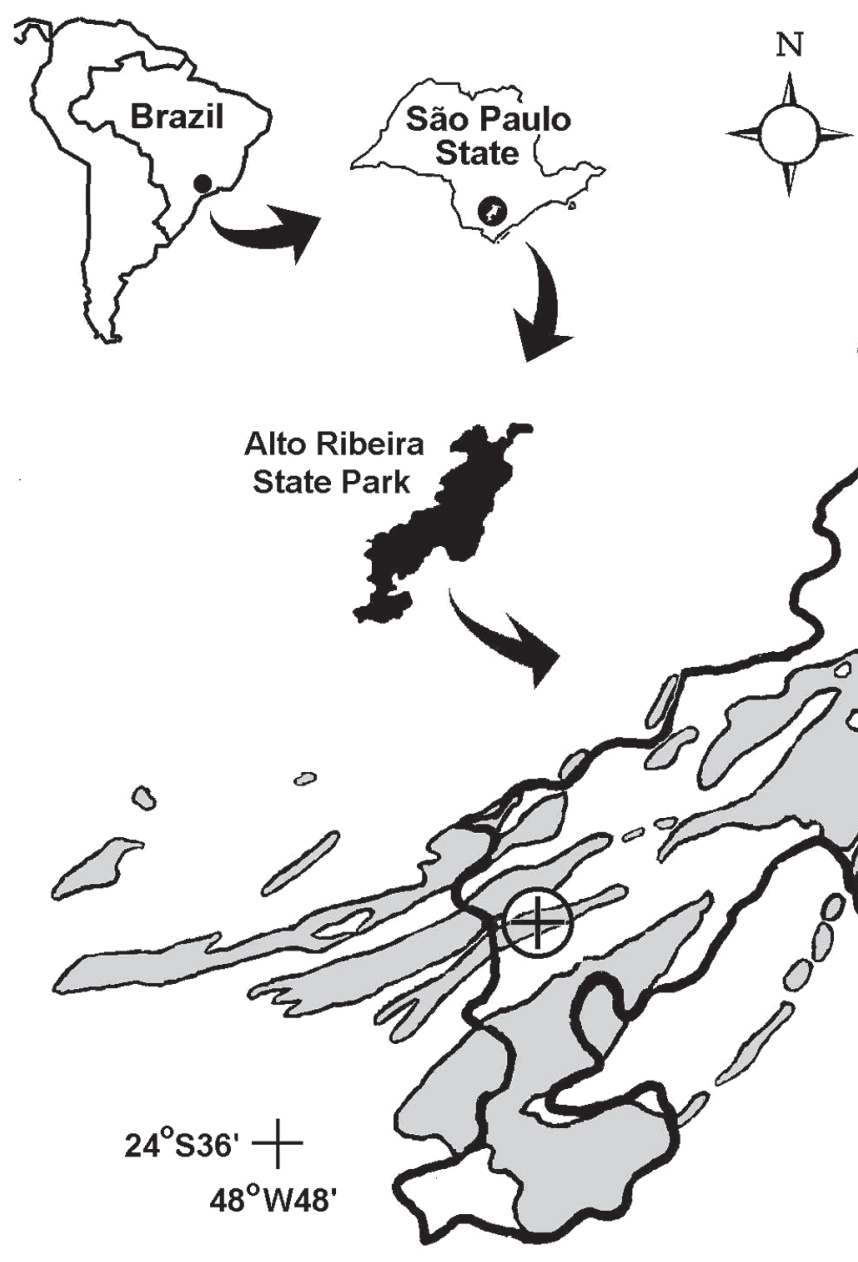

Figure 1. Localization of the study area and occurrence of calcareous soil.

Forest survey - The study area was chosen through field observations, interviews with the farmer who abandoned the crop areas, analysis of aerial photographs from 1962, 1973, 1981 and 1997 (figures 2-5), and LANDSAT TM 5 orbital image of 1990. Scales varied between 1:25000 and 1:50 000. The phytossociological survey was carried out in three different adjacent spots corresponding to different times of abandonment after slash-and-burn agriculture: Phase I (PI) with 15 years; Phase II (PII) with 25 years; and Phase III (PIII) with more than 36 years without disturbance and probably never clear-cut (figure 2). The inventory was made using transects $50 \mathrm{~m}$ long and $20 \mathrm{~m}$ wide $(0.1 \mathrm{ha})$. The inclusion criterion was trunk perimeter at breast height $\geq 15$ $\mathrm{cm}$. All individuals were labelled, measured in height and perimeter, mapped and sampled for taxonomic identification. The vouchers were deposited at the Herbarium of São Paulo Forestry Institute (SPSF). Tree heights (m) were measured by an opti-meter Ranging 120, Ranging Inc. Species binomials were standardized according to Missouri Botanical Garden (http://mobot.org/ W3T/Search/vast.html) and New York
Botanical Garden (http://www. nybg.org/search).

The Relative Dominance (RDo) is an index to assess relative space occupation and is estimated by the relative basal area of all individuals belonging to one species or family. The basal area was calculated by converting perimeter to basal area in $\mathrm{cm}^{2}$. The Species Relative Density (RDi) is an index to assess the species relative distribution. The sum of these two parameters gives the Coverage Index for each Species (CIi) (Müller-Dombois et al. 1974).

$\mathrm{RDoi}=(\mathrm{BAi} \times 100) / \Sigma \mathrm{BAn}$

$\mathrm{RDi}=(\mathrm{Ni} \times 100) / \mathrm{Nm}$

$\mathrm{Cli}=\mathrm{RDoi}+\mathrm{RDi}$

Where:

RDoi - Species Relative Dominance

BAi - Species Basal Area

¿BAn - Total Basal Area

RDi - Species Relative Density

$\mathrm{Ni}$ - Species Number of individuals

$\mathrm{Nn}$ - Total Number of individuals

CIi - Species Coverage Index 


\section{Results}

The analysis of historical series of aerial photography from the study site was critical for definition of the age of each forest fragment studied, i.e. the time elapsed from the abandonment of the crop field, when it occurred. For the forest fragment called PIII, it was possible to determine that it was not cut since 1962, and probably long before that, as we could see from the size of trees occuring at that time, resulting in an estimate of more than 36 years in 1998. With respect to the fragment called PII, it was possible to define the time elapsed after the area had been abandoned, corresponding to aproximadetely 25 years in 1998. For the fragment called PI, the time elapsed after the abandonment of the crop field was estimated through interviews with the farmer and by analysis of aerial photography from 1981 (not shown),
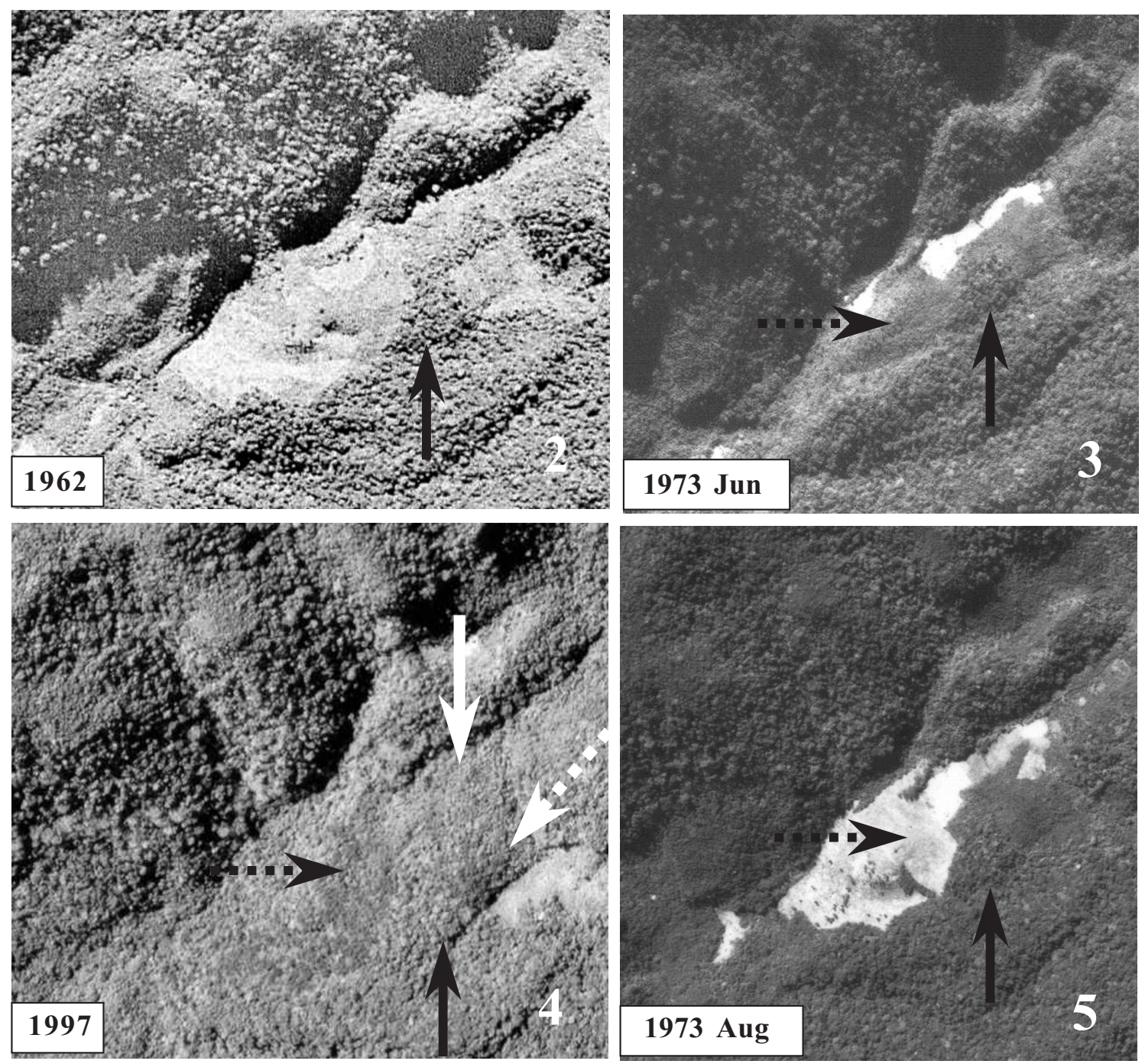

Figure 2-5. Historical series of aerial photography showing the evolution of the vegetation cover in the study area. 2. Aerial photo (1962) showing a forested area, which remains uncut (black arrow) throughout the time comprehended by our study. 3 and 5. Aerial photos from two different periods in 1973 (June and August, respectively) showing a forest fragment cut (dotted arrow) and the uncut fragment (black arrow). 4. Aerial photo (1997) showing the successional phases inventoried: white arrow-phase I (15 years after abandonment); white dotted arrow-phase II (25 years); black arrow-phase III (36 + years); the black dotted arrow indicates a regeneration area dominated by Piptadenia gonoacantha, similar to the area where phase II was inventoried. 
and resulted in approximately 15 years.

Considering the complete survey, including all areas studied, were registered 34 families (two unidentified), 75 genera (seven unidentified), and 87 species (six unidentified) (table 1).

The phytossociological survey indicated a decrease in tree density along succession: PI - 1940 ind.ha' ${ }^{-1}$; PII - 1690 ind.ha ${ }^{-1}$; and PIII - 1520 ind.ha ${ }^{-1}$. The Species Relative Dominance (RDoi) and Coverage Index (Cli) indicates that, besides a single exception (Myrsine coriacea; CIi), top positions are occupied by species belonging to Leguminosae (table 1, figure $6)$.

PI is dominated by Piptadenia gonoacantha and Symplocos laxiflora in respect to RDoi. The CIi follows the RDo pattern, with P. gonoacantha as the main species (Figure 6). In this area, 38 species (two unidentified) belonging to 33 genus (one unidentified) and 21 families were sampled. The family Leguminosae was the most important with respect to Relative Dominance, Coverage Index, number of individuals $\left(\mathrm{n}_{\mathrm{i}}=48\right)$ and number of species $\left(\mathrm{n}_{\mathrm{sp}}=8\right)$, following by Lauraceae $\left(\mathrm{n}_{\mathrm{i}}=13 ; \mathrm{n}_{\mathrm{sp}}=6\right)$ and Annonaceae $\left(\mathrm{n}_{\mathrm{i}}=22 ; \mathrm{n}_{\mathrm{sp}}=4\right)$ (figure 7$)$.

PII is dominated by $P$. gonoacantha and $M$. coriacea, both with increased RDoi related to the former stage. Here, the CIi indicates that M. coriacea showed a slightly higher value, which is correlated with its higher number of individuals that occur mainly in the forest understorey (figure 6). In this stage, 29 (three unidentified) species, 28 genus and 15 families were sampled, which represents a clear decrease in family and species diversity. The family Leguminosae was the first in importance with respect to number of species $\left(\mathrm{n}_{\mathrm{sp}}=7\right)$ and Relative Dominance, and second with respect to number of individuals $\left(n_{i}=25\right)$ and Coverage Index. The family Myrsinaceae was the first with respect to number of individuals $\left(n_{i}=43\right)$ and because of that, also with respect to Coverage Index. It is important to notice that this latter family was represented by only one species, which is typical of the understorey habitat. Melastomataceae $\left(\mathrm{n}_{\mathrm{i}}=18\right.$; $\left.\mathrm{n}_{\mathrm{sp}}=4\right)$, Piperaceae $\left(\mathrm{n}_{\mathrm{i}}=18 ; \mathrm{n}_{\mathrm{sp}}=2\right)$ and Euphorbiaceae $\left(\mathrm{n}_{\mathrm{i}}=11 ; \mathrm{n}_{\mathrm{sp}}=3\right)$ were also important (figure 7).

PIII is dominated by Schizolobium parahyba, but its RDo is lower than that observed for the dominant species in PI and PII. Actually, some species can be seen with almost the same importance, like Hymenaea courbaril, Ficus sp., Guapira opposita and Aspidosperma ramiflorum (figure 6). This reflects the higher diversity of PIII, where 58 species belonging to 53 genus (six unidentified) and 23 families (two unidentified) were sampled. CIi shows a slightly different pattern, where understorey species like Euterpe edulis and Guapira opposita, assumed higher importance because of higher number of individuals. Myrtaceae was the most important family with respect to number of individuals and species $\left(\mathrm{n}_{\mathrm{i}}=23 ; \mathrm{n}_{\mathrm{sp}}=11\right)$. The Leguminosae was the most important with respect to Relative Dominance, and ranked second with respect to number of species

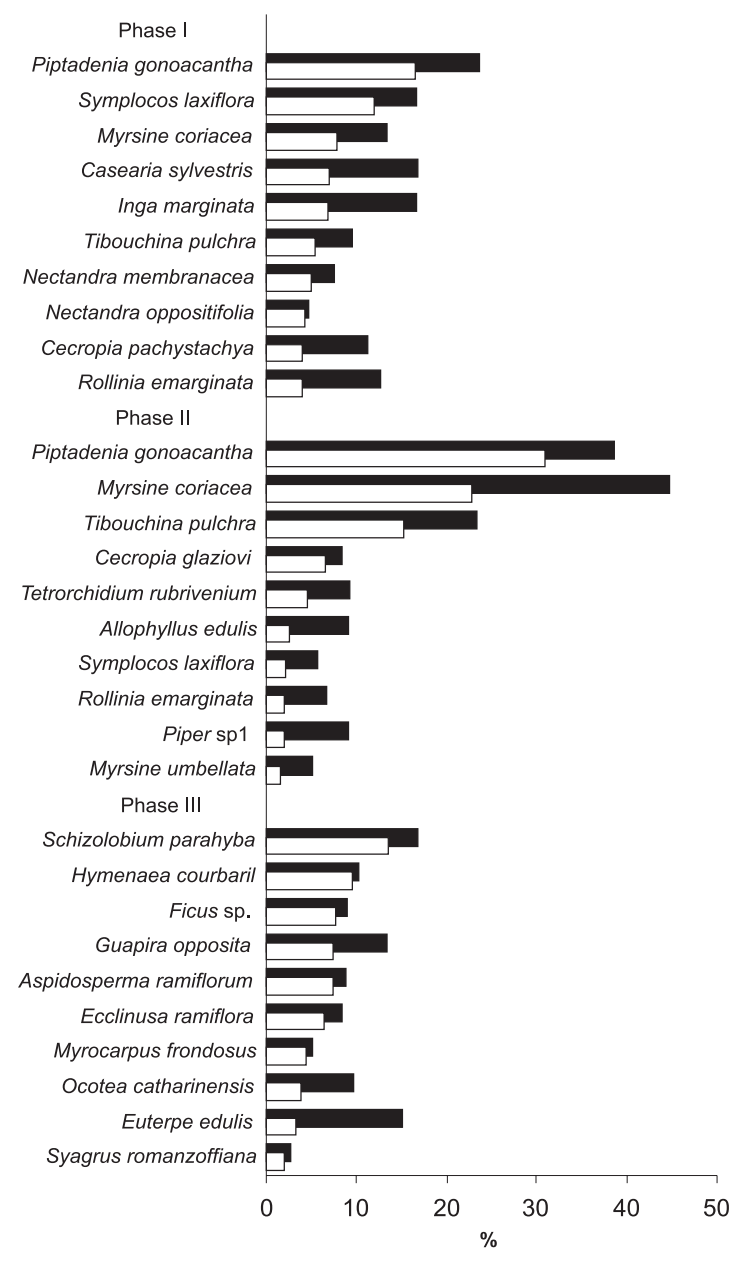

Figure 6. Distribution of the parameters - Coverage Index ( CIi) and Relative Dominance ( $\square$ RDoi) among the species inventoried in the different successional phases. PI - phase I (15 years after abandonment of crop field); PII - phase II (25 years); PIII - phase III (36 + years). 
Table 1. Occurrence of families and species in the successional phases sampled over calcareous soils at the Alto Ribeira Touristic State Park - PETAR, SP. Phase I - 15 years after abandonment of crop field; Phase II - 25 years; Phase III - $36+$ years.

\begin{tabular}{l|ll}
\hline Family/Species & Phase I & Phase II \\
\hline
\end{tabular}

\section{ANNONACEAE}

Guatteria nigrescens Mart.

Rollinia sericea (R.E. Fr.) R.E. Fr.

Rollinia emarginata Schltdl.

Annonaceae sp. 1

Annonaceae sp.4

Annonaceae sp.5

$\mathrm{X}$

$\mathrm{X}$

$\mathrm{X}$

$\mathrm{X}$

X

$\mathrm{X}$

$\mathrm{X}$

APOCYNACEAE

Aspidosperma polyneuron Müll. Arg.

Aspidosperma ramiflorum Müll. Arg.

$\mathrm{X}$

$\mathrm{X}$

\section{ARECACEAE}

Euterpe edulis Mart.

Syagrus romanzoffiana (Cham.) Glassman

\section{ASTERACEAE}

Verbesina sp.

BORAGINACEAE

Cordia trichotoma (Vell.) Arráb. ex Steud.

BURSERACEAE

Protium heptaphyllum (Aubl.) Marchand

CECROPIACEAE

Cecropia glaziovi Snethlage

Cecropia pachystachya Trécul

Coussapoa microcarpa (Schott) Rizzini

CELASTRACEAE

Maytenus cf. evonymoides Reisseck

CLETRACEAE

Clethra scabra Pers.

CLUSIACEAE

Garcinia gardneriana (Planch. \& Triana) Zappi

EUPHORBIACEAE

Alchornea triplinervia (Spreng.) Müll. Arg.

Hyeronima alchorneoides Allemao

Margaritaria nobilis L.f.

Tetrorchidium rubrivenium Poepp.

\section{FLACOURTIACEAE}

Casearia sylvestris $\mathrm{Sw}$.

\section{LAURACEAE}

Beilschmiedia emarginata (Nees) Kosterm. Endlicheria paniculata (Spreng.) J.F. Macbr. Nectandra megapotamica (Sprengel) Mez Nectandra membranacea (Sw.) Griseb. Nectandra oppositifolia Nees \& Mart.
$\mathrm{X}$

X

$\mathrm{X}$

$\mathrm{X}$

$\mathrm{X}$

X

X

X

X

X

$\mathrm{X}$

X

$\mathrm{X}$

$\begin{array}{ccc}X & X & X \\ & X & \\ & & X \\ X & X & X\end{array}$

X

$\mathrm{X}$

X

$\mathrm{X}$

$\mathrm{X}$ 


\begin{tabular}{|c|c|c|c|}
\hline Family/Species & Phase I & Phase II & Phase III \\
\hline Ocotea catharinensis $\mathrm{Mez}$ & & & $\mathrm{X}$ \\
\hline Ocotea sp. & $\mathrm{X}$ & & \\
\hline Persea venosa Nees \& Mart. ex Nees & $\mathrm{X}$ & & $\mathrm{X}$ \\
\hline \multicolumn{4}{|l|}{ LEGUMINOSAE } \\
\hline Bauhinia forficata Link & $\mathrm{X}$ & $\mathrm{X}$ & \\
\hline Dalbergia frutescens (Vell.) Britton & & $\mathrm{X}$ & \\
\hline Hymenaea courbaril L. & & & $\mathrm{X}$ \\
\hline Inga marginata Willd. & $\mathrm{X}$ & $\mathrm{X}$ & $\mathrm{X}$ \\
\hline Lonchocarpus muehlbergianus Hassl. & & & $\mathrm{X}$ \\
\hline Machaerium nyctitans (Vell.) Benth. & $\mathrm{X}$ & & \\
\hline Machaerium lanatum Tul. & $\mathrm{X}$ & & \\
\hline Machaerium stipitatum (DC.) Vogel & & $\mathrm{X}$ & $\mathrm{X}$ \\
\hline Myrocarpus frondosus Allemao & & & $\mathrm{X}$ \\
\hline Piptadenia gonoacantha (Mart.) J.F. Macbr. & $\mathrm{X}$ & $\mathrm{X}$ & $\mathrm{X}$ \\
\hline Piptadenia paniculata Benth. & $\mathrm{X}$ & $\mathrm{X}$ & \\
\hline Platymiscium floribundum Vogel & $\mathrm{X}$ & $\mathrm{X}$ & \\
\hline Pterocarpus cf. rohrii Vahl & & & $\mathrm{X}$ \\
\hline Schizolobium parahyba (Vell.) S.F. Blake & $\mathrm{X}$ & & $\mathrm{X}$ \\
\hline Swartzia aff. acutifolia Vogel & & & $\mathrm{X}$ \\
\hline \multicolumn{4}{|l|}{ MALPIGHIACEAE } \\
\hline Byrsonima ligustrifolia A. Juss. & & $\mathrm{X}$ & $\mathrm{X}$ \\
\hline \multicolumn{4}{|l|}{ MELASTOMATACEAE } \\
\hline Leandra mosenii Cogn. & & $\mathrm{X}$ & \\
\hline Miconia cinnamomifolia (DC.) Naudin & & $\mathrm{X}$ & \\
\hline Miconia latecrenata Triana & & $\mathrm{X}$ & \\
\hline Tibouchina pulchra (Cham.) Cogn. & $\mathrm{X}$ & $\mathrm{X}$ & \\
\hline Melastomataceae sp. 1 & & & $\mathrm{X}$ \\
\hline \multicolumn{4}{|l|}{ MELIACEAE } \\
\hline Cedrela fissilis Vell. & & & $\mathrm{X}$ \\
\hline Guarea macrophylla Vahl & & & $\mathrm{X}$ \\
\hline Trichilia lepidota Mart. & & & $\mathrm{X}$ \\
\hline \multicolumn{4}{|l|}{ MONIMIACEAE } \\
\hline Mollinedia clavigera Tul. & & & $\mathrm{X}$ \\
\hline Monimiaceae sp.1 & & & $\mathrm{X}$ \\
\hline \multicolumn{4}{|l|}{ MORACEAE } \\
\hline Ficus sp. & & & $\mathrm{X}$ \\
\hline Sorocea bonplandii (Baill.) W.C. Burger, Lanj. \& Wess. Boer & & & $\mathrm{X}$ \\
\hline Brosimum guianense (Aubl.) Huber & & & $\mathrm{X}$ \\
\hline \multicolumn{4}{|l|}{ MYRSINACEAE } \\
\hline Myrsine coriacea (Sw.) R. Br. ex Roem. \& Schult. & $\mathrm{X}$ & $\mathrm{X}$ & \\
\hline Myrsine umbellata Mart. & & $\mathrm{X}$ & \\
\hline \multicolumn{4}{|l|}{ MYRTACEAE } \\
\hline Calyptranthes concinna DC. & & & $\mathrm{X}$ \\
\hline Campomanesia guaviroba (DC.) Kiaerskow & $\mathrm{X}$ & $\mathrm{X}$ & $\mathrm{X}$ \\
\hline Eugenia cuprea $(\mathrm{O}$. Berg) Mattos & & & $\mathrm{X}$ \\
\hline Eugenia florida DC. & & & $\mathrm{X}$ \\
\hline Eugenia riedeliana $\mathrm{O}$. Berg & & & $\mathrm{X}$ \\
\hline Gomidesia tijucensis (Kiaersk.) D. Legrand & & & $\mathrm{X}$ \\
\hline Marlierea eugeniopsoides (D. Legrand \& Kausel) D. Legrand & & & $\mathrm{X}$ \\
\hline Myrceugenia myrcioides (Cambess.) O. Berg & & & $\mathrm{X}$ \\
\hline
\end{tabular}




\begin{tabular}{|c|c|c|c|}
\hline Family/Species & Phase I & Phase II & Phase III \\
\hline Myrcia formosiana DC. & $\mathrm{X}$ & $\mathrm{X}$ & \\
\hline Myrcia pubipetala Miq. & & & $\mathrm{X}$ \\
\hline Psidium cattleyanum Sabine & & $\mathrm{X}$ & \\
\hline Myrtaceae sp.1 & & & $\mathrm{X}$ \\
\hline Myrtaceae sp. 3 & & & $\mathrm{X}$ \\
\hline \multicolumn{4}{|l|}{ NYCTAGINACEAE } \\
\hline Guapira opposita (Vell.) Reitz & $\mathrm{X}$ & & $\mathrm{x}$ \\
\hline \multicolumn{4}{|l|}{ OLACACEAE } \\
\hline Heisteria silvianii Schwacke & $\mathrm{X}$ & & \\
\hline \multicolumn{4}{|l|}{ PIPERACEAE } \\
\hline Piper sp.1 & & $\mathrm{X}$ & \\
\hline Piper sp.2 & & $\mathrm{X}$ & \\
\hline \multicolumn{4}{|l|}{ PROTEACEAE } \\
\hline Roupala brasiliensis Klotzsch & & & $\mathrm{X}$ \\
\hline \multicolumn{4}{|l|}{ QUIINACEAE } \\
\hline Quiina glaziovii Engl. & $\mathrm{X}$ & & \\
\hline \multicolumn{4}{|l|}{ ROSACEAE } \\
\hline Prunus myrtifolia (L.) Urb. & & & $\mathrm{x}$ \\
\hline \multicolumn{4}{|l|}{ RUBIACEAE } \\
\hline Alibertia myrciifolia Schum. & & & $\mathrm{X}$ \\
\hline Alseis floribunda Schott & $\mathrm{X}$ & & $\mathrm{X}$ \\
\hline Bathysa meridionalis Smith \& Downs & & $\mathrm{X}$ & \\
\hline Posoqueria acutifolia Mart. & & & $\mathrm{X}$ \\
\hline Psychotria cf. carthagenensis Jacq. & & $\mathrm{X}$ & \\
\hline Rudgea cf. jasminoides (Cham.) Müll. Arg. & & & $\mathrm{X}$ \\
\hline \multicolumn{4}{|l|}{ SABIACEAE } \\
\hline Meliosma sellowii Urb. & $\mathrm{X}$ & & \\
\hline \multicolumn{4}{|l|}{ SAPINDACEAE } \\
\hline Allophyllus edulis (A.St. Hil.) Radlk. & $\mathrm{X}$ & $\mathrm{X}$ & $\mathrm{X}$ \\
\hline Cupania oblongifolia Mart. & $\mathrm{X}$ & $\mathrm{X}$ & \\
\hline Matayba guianensis Aubl. & $\mathrm{X}$ & & \\
\hline Matayba juglandifolia (Cambess.) Radlk. & & & $\mathrm{x}$ \\
\hline \multicolumn{4}{|l|}{ SAPOTACEAE } \\
\hline Chrysophyllum inornatum Mart. & & & $\mathrm{X}$ \\
\hline Ecclinusa ramiflora Mart. & & & $\mathrm{X}$ \\
\hline Pouteria psammophila (Mart.) Radlk. & $\mathrm{X}$ & & $\mathrm{X}$ \\
\hline \multicolumn{4}{|l|}{ SYMPLOCACEAE } \\
\hline Symplocos laxiflora Benth. & $\mathrm{X}$ & $\mathrm{X}$ & \\
\hline \multicolumn{4}{|l|}{ ULMACEAE } \\
\hline Trema micrantha (L.) Blume & $\mathrm{X}$ & $\mathrm{X}$ & \\
\hline \multicolumn{4}{|l|}{ VERBENACEAE } \\
\hline Aegiphila sp. & $\mathrm{X}$ & & \\
\hline \multicolumn{4}{|l|}{ UNIDENTIFIED } \\
\hline Sp.1 & & & $\mathrm{X}$ \\
\hline Sp.3 & & & $\mathrm{X}$ \\
\hline
\end{tabular}




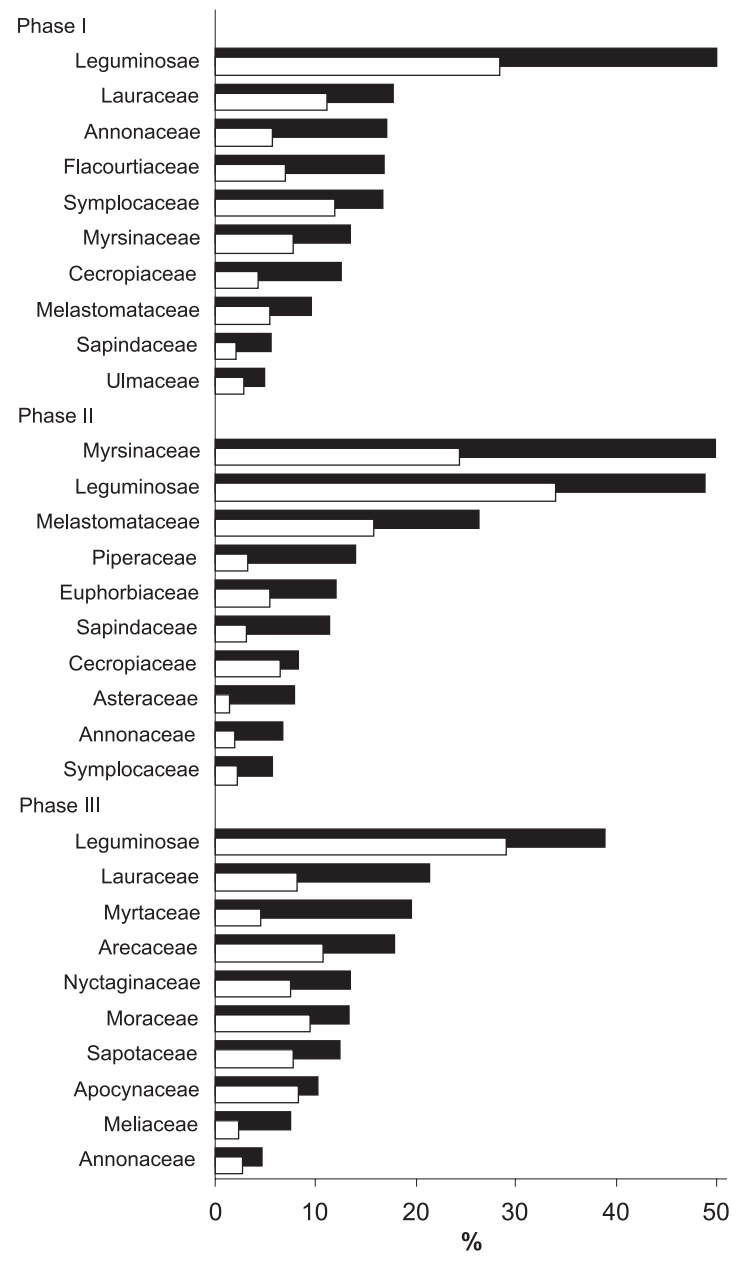

Figure 7. Distribution of the parameters - Coverage Index ( $\square$ CIi) and Relative Dominance ( $\square$ RDoi) among the angiosperm families inventoried in the different successional phases. PI phase I (15 years after abandonment of crop field); PII phase II (25 years); PIII - phase III (36 + years).

$\left(\mathrm{n}_{\mathrm{i}}=15 ; \mathrm{n}_{\mathrm{sp}}=9\right)$. Lauraceae $\left(\mathrm{n}_{\mathrm{i}}=20 ; \mathrm{n}_{\mathrm{sp}}=7\right)$ and Arecaceae $\left(\mathrm{n}_{\mathrm{i}}=19 ; \mathrm{n}_{\mathrm{sp}}=2\right)$ were also important (figure 7).

Considering all inventories, the family Leguminosae was the most important in all aspects investigated ( $\mathrm{n}^{\circ}$ of individuals and species; Relative Dominance; Coverage Index). Other important families were Myrsinaceae, Myrtaceae, Annonaceae, Lauraceae and Melastomataceae (figure 8).

\section{Discussion}

The analysis of aerial photography was decisive to define the age of forest fragments in the study
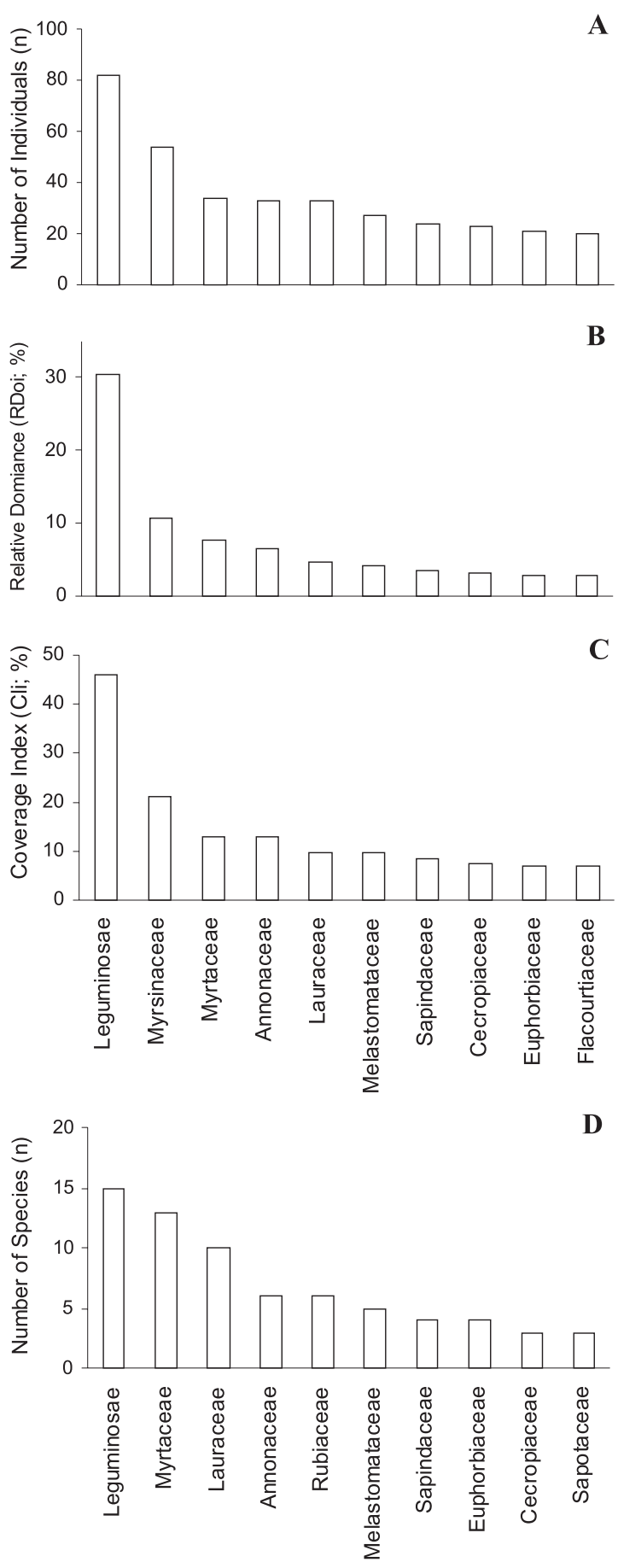

Figure 8. Distribution of some parameters among the angiosperm families inventoried, considering all successional phases together. A. Number of Individuals (n); B. Relative Dominance (RDoi) (\%); C. Coverage Index (CIi) (\%); D. Number of Species (n). 
Table 2. Phytosociological survey in the three different phases of succession over calcareous soil - PETAR. Phase I - 15 years after abandonment of crop field; Phase II - 25 years; Phase III - 36 + years; RDi - species Relative Density; RDoi - species Relative Dominance; CIi - species Coverage Index; Ni - species number of individuals.

\begin{tabular}{|c|c|c|c|c|c|}
\hline Species & Family & $\mathrm{Ni}$ & RDoi & RDi & CIi \\
\hline \multicolumn{6}{|l|}{ Phase I } \\
\hline Piptadenia gonoacantha & Leguminosae & 14 & 16.5 & 7.2 & 23.7 \\
\hline Dead & & 19 & 7.8 & 9.8 & 17.6 \\
\hline Casearia sylvestris & Flacourtiaceae & 19 & 7.0 & 9.8 & 16.8 \\
\hline Inga marginata & Leguminosae & 19 & 6.9 & 9.8 & 16.7 \\
\hline Symplocos laxiflora & Symplocaceae & 9 & 11.9 & 4.6 & 16.6 \\
\hline Myrsine coriacea & Myrsinaceae & 11 & 7.8 & 5.7 & 13.4 \\
\hline Rollinia emarginata & Annonaceae & 17 & 3.9 & 8.8 & 12.7 \\
\hline Cecropia pachystachya & Cecropiaceae & 14 & 4.0 & 7.2 & 11.2 \\
\hline Tibouchina pulchra & Melastomataceae & 8 & 5.4 & 4.1 & 9.5 \\
\hline Nectandra membranacea & Lauraceae & 5 & 4.9 & 2.6 & 7.5 \\
\hline Trema micrantha & Ulmaceae & 4 & 2.8 & 2.1 & 4.9 \\
\hline Nectandra oppositifolia & Lauraceae & 1 & 4.2 & 0.5 & 4.7 \\
\hline Bauhinia forficata & Leguminosae & 4 & 2.0 & 2.1 & 4.1 \\
\hline Matayba guianensis & Sapindaceae & 3 & 1.5 & 1.5 & 3.0 \\
\hline Myrcia formosiana & Myrtaceae & 3 & 0.9 & 1.5 & 2.4 \\
\hline Tetrorchidium rubrivenium & Euphorbiaceae & 2 & 1.1 & 1.0 & 2.1 \\
\hline Cordia trichotoma & Boraginaceae & 3 & 0.5 & 1.5 & 2.1 \\
\hline Nectandra megapotamica & Lauraceae & 3 & 0.5 & 1.5 & 2.0 \\
\hline Campomanesia guaviroba & Myrtaceae & 3 & 0.4 & 1.5 & 2.0 \\
\hline Guatteria nigrescens & Annonaceae & 3 & 0.4 & 1.5 & 2.0 \\
\hline Cupania oblongifolia & Sapindaceae & 3 & 0.4 & 1.5 & 1.9 \\
\hline Schizolobium parahyba & Leguminosae & 1 & 1.4 & 0.5 & 1.9 \\
\hline Alchornea triplinervia & Euphorbiaceae & 3 & 0.3 & 1.5 & 1.8 \\
\hline Endlicheria paniculata & Lauraceae & 2 & 0.5 & 1.0 & 1.5 \\
\hline Annonaceae sp.1 & Annonaceae & 1 & 1.0 & 0.5 & 1.5 \\
\hline Meliosma sellowii & Sabiaceae & 2 & 0.5 & 1.0 & 1.5 \\
\hline Pouteria psammophila & Sapotaceae & 2 & 0.5 & 1.0 & 1.5 \\
\hline Cecropia glaziovi & Cecropiaceae & 2 & 0.3 & 1.0 & 1.3 \\
\hline Piptadenia paniculata & Leguminosae & 1 & 0.6 & 0.5 & 1.1 \\
\hline Persea venosa & Lauraceae & 1 & 0.6 & 0.5 & 1.1 \\
\hline Platymiscium floribundum & Leguminosae & 1 & 0.5 & 0.5 & 1.0 \\
\hline Aegiphila sp. & Verbenaceae & 1 & 0.5 & 0.5 & 1.0 \\
\hline Rollinia sericea & Annonaceae & 1 & 0.4 & 0.5 & 0.9 \\
\hline Machaerium lanatum & Leguminosae & 1 & 0.4 & 0.5 & 0.9 \\
\hline Ocotea sp. & Lauraceae & 1 & 0.4 & 0.5 & 0.9 \\
\hline Clethra scabra & Clethraceae & 1 & 0.4 & 0.5 & 0.9 \\
\hline Heisteria silvianii & Olacaceae & 1 & 0.3 & 0.5 & 0.9 \\
\hline Alseis floribunda & Rubiaceae & 1 & 0.2 & 0.5 & 0.7 \\
\hline Guapira opposita & Nyctaginaceae & 1 & 0.2 & 0.5 & 0.7 \\
\hline Allophyllus edulis & Sapindaceae & 1 & 0.2 & 0.5 & 0.7 \\
\hline Quiina glaziovii & Quiinaceae & 1 & 0.1 & 0.5 & 0.6 \\
\hline Machaerium nyctitans & Leguminosae & 1 & 0.1 & 0.5 & 0.6 \\
\hline \multicolumn{6}{|l|}{ Phase II } \\
\hline Myrsine coriacea & Myrsinaceae & 37 & 22.8 & 21.9 & 44.7 \\
\hline Piptadenia gonoacantha & Leguminosae & 13 & 30.9 & 7.7 & 38.6 \\
\hline Tibouchina pulchra & Melastomataceae & 14 & 15.2 & 8.3 & 23.4 \\
\hline Tetrorchidium rubrivenium & Euphorbiaceae & 8 & 4.5 & 4.7 & 9.3 \\
\hline Allophyllus edulis & Sapindaceae & 11 & 2.6 & 6.5 & 9.1 \\
\hline Piper sp. 1 & Piperaceae & 12 & 1.9 & 7.1 & 9.0 \\
\hline Cecropia glaziovi & Cecropiaceae & 3 & 6.5 & 1.8 & 8.3 \\
\hline Verbesina $\mathrm{sp}$ & Asteraceae & 11 & 1.4 & 6.5 & 7.9 \\
\hline Rollinia emarginata & Annonaceae & 8 & 2.0 & 4.7 & 6.7 \\
\hline
\end{tabular}


(cont.)

\begin{tabular}{|c|c|c|c|c|c|}
\hline Species & Family & $\mathrm{Ni}$ & RDoi & RDi & CIi \\
\hline Symplocos laxiflora & Symplocaceae & 6 & 2.2 & 3.6 & 5.7 \\
\hline Myrsine umbellata & Myrsinaceae & 6 & 1.5 & 3.6 & 5.1 \\
\hline Piper sp.2 & Piperaceae & 6 & 1.3 & 3.6 & 4.9 \\
\hline Piptadenia paniculata & Leguminosae & 4 & 0.9 & 2.4 & 3.3 \\
\hline Cupania oblongifolia & Sapindaceae & 3 & 0.5 & 1.8 & 2.3 \\
\hline Inga marginata & Leguminosae & 2 & 0.9 & 1.2 & 2.1 \\
\hline Campomanesia guaviroba & Myrtaceae & 2 & 0.8 & 1.2 & 1.9 \\
\hline Bauhinia forficata & Leguminosae & 2 & 0.6 & 1.2 & 1.8 \\
\hline Machaerium stipitatum & Leguminosae & 2 & 0.4 & 1.2 & 1.6 \\
\hline Miconia cinnamomifolia & Melastomataceae & 2 & 0.4 & 1.2 & 1.5 \\
\hline Dead & & 2 & 0.3 & 1.2 & 1.5 \\
\hline Hyeronima alchorneoides & Euphorbiaceae & 2 & 0.2 & 1.2 & 1.4 \\
\hline Psidium cattleyanum & Myrtaceae & 2 & 0.2 & 1.2 & 1.4 \\
\hline Alchornea triplinervia & Euphorbiaceae & 1 & 0.7 & 0.6 & 1.3 \\
\hline Casearia sylvestris & Flacourtiaceae & 1 & 0.3 & 0.6 & 0.8 \\
\hline Trema micrantha & Ulmaceae & 1 & 0.2 & 0.6 & 0.8 \\
\hline Bathysa meridionalis & Rubiaceae & 1 & 0.2 & 0.6 & 0.8 \\
\hline Myrcia formosiana & Myrtaceae & 1 & 0.2 & 0.6 & 0.8 \\
\hline Leandra mosenii & Melastomataceae & 1 & 0.1 & 0.6 & 0.7 \\
\hline Miconia latecrenata & Melastomataceae & 1 & 0.1 & 0.6 & 0.7 \\
\hline Byrsonima ligustrifolia & Malpiguiaceae & 1 & 0.1 & 0.6 & 0.7 \\
\hline Psychotria cf. carthagenensis & Rubiaceae & 1 & 0.1 & 0.6 & 0.7 \\
\hline Dalbergia frutescens & Leguminosae & 1 & 0.1 & 0.6 & 0.7 \\
\hline Platymiscium floribundum & Leguminosae & 1 & 0.1 & 0.6 & 0.7 \\
\hline \multicolumn{6}{|l|}{ Phase III } \\
\hline Schizolobium parahyba & Leguminosae & 5 & 13.47 & 3.3 & 16.8 \\
\hline Hymenaea courbaril & Leguminosae & 1 & 9.58 & 0.7 & 10.2 \\
\hline Ficus sp. & Moraceae & 2 & 7.69 & 1.3 & 9 \\
\hline Guapira opposita & Nyctaginaceae & 9 & 7.45 & 5.9 & 13.4 \\
\hline Aspidosperma ramiflorum & Apocynaceae & 2 & 7.43 & 1.3 & 8.7 \\
\hline Ecclinusa ramiflora & Sapotaceae & 3 & 6.44 & 2 & 8.4 \\
\hline Myrocarpus frondosus & Leguminosae & 1 & 4.44 & 0.7 & 5.1 \\
\hline Ocotea catharinensis & Lauraceae & 9 & 3.77 & 5.9 & 9.7 \\
\hline Euterpe edulis & Arecaceae & 18 & 3.23 & 11.8 & 15.1 \\
\hline Syagrus romanzoffiana & Arecaceae & 1 & 2.05 & 0.7 & 2.7 \\
\hline Dead & & 3 & 2 & 2 & 4 \\
\hline Melastomataceae sp.1 & Melastomataceae & 1 & 1.93 & 0.7 & 2.6 \\
\hline Endlicheria paniculata & Lauraceae & 4 & 1.64 & 2.6 & 4.3 \\
\hline Allophyllus edulis & Sapindaceae & 2 & 1.64 & 1.3 & 3 \\
\hline Nectandra megapotamica & Lauraceae & 2 & 1.66 & 1.3 & 3 \\
\hline Myrtaceae sp.3 & Myrtaceae & 1 & 1.55 & 0.7 & 2.2 \\
\hline Protium heptaphyllum & Burseraceae & 1 & 1.52 & 0.7 & 2.2 \\
\hline Trichilia lepidota & Meliaceae & 3 & 1.23 & 2 & 3.2 \\
\hline Roupala brasiliensis & Proteaceae & 1 & 1.22 & 0.7 & 1.9 \\
\hline Coussapoa microcarpa & Cecropiaceae & 2 & 1.22 & 1.3 & 2.5 \\
\hline Brosimum guianense & Moraceae & 2 & 1.18 & 1.3 & 2.5 \\
\hline Annonaceae sp.5 & Annonaceae & 1 & 1.12 & 0.7 & 1.8 \\
\hline Prunus myrtifolia & Rosaceae & 2 & 1.11 & 1.3 & 2.4 \\
\hline Maytenus cf. evonymoides & Celastraceae & 3 & 0.98 & 2 & 3 \\
\hline Piptadenia gonoacantha & Leguminosae & 1 & 0.97 & 0.7 & 1.6 \\
\hline Annonaceae sp.4 & Annonaceae & 1 & 0.92 & 0.7 & 1.6 \\
\hline Tetrorchidium rubrivenium & Euphorbiaceae & 2 & 0.88 & 1.3 & 2.2 \\
\hline Cedrela fissilis & Meliaceae & 3 & 0.87 & 2 & 2.8 \\
\hline Chrysophyllum inornatum & Sapotaceae & 3 & 0.82 & 2 & 2.8 \\
\hline Aspidosperma polyneuron & Apocynaceae & 1 & 0.8 & 0.7 & 1.5 \\
\hline Rollinia sericea & Annonaceae & 1 & 0.64 & 0.7 & 1.3 \\
\hline Margaritaria nobilis & Euphorbiaceae & 2 & 0.6 & 1.3 & 1.9 \\
\hline
\end{tabular}


(cont.)

\begin{tabular}{|c|c|c|c|c|c|}
\hline Species & Family & $\mathrm{Ni}$ & RDoi & RDi & CIi \\
\hline Pouteria psammophila & Sapotaceae & 1 & 0.53 & 0.7 & 1.2 \\
\hline Campomanesia guaviroba & Myrtaceae & 2 & 0.53 & 1.3 & 1.8 \\
\hline Sorocea bonplandii & Moraceae & 2 & 0.51 & 1.3 & 1.8 \\
\hline Persea venosa & Lauraceae & 1 & 0.47 & 0.7 & 1.1 \\
\hline Myrceugenia myrcioides & Myrtaceae & 4 & 0.43 & 2.6 & 3.1 \\
\hline Eugenia cuprea & Myrtaceae & 5 & 0.42 & 3.3 & 3.7 \\
\hline Nectandra oppositifolia & Lauraceae & 1 & 0.38 & 0.7 & 1 \\
\hline Gomidesia tijucensis & Myrtaceae & 1 & 0.38 & 0.7 & 1 \\
\hline Marlierea eugeniopsoides & Myrtaceae & 3 & 0.36 & 2 & 2.3 \\
\hline Myrcia pubipetala & Myrtaceae & 3 & 0.33 & 2 & 2.3 \\
\hline Verbesina sp. & Asteraceae & 3 & 0.3 & 2 & 2.3 \\
\hline Alibertia myrciifolia & Rubiaceae & 2 & 0.3 & 1.3 & 1.6 \\
\hline Garcinia gardneriana & Clusiaceae & 2 & 0.25 & 1.3 & 1.6 \\
\hline Cecropia pachystachya & Cecropiaceae & 2 & 0.23 & 1.3 & 1.5 \\
\hline Posoqueria acutifolia & Rubiaceae & 1 & 0.21 & 0.7 & 0.9 \\
\hline Matayba junglandifolia & Sapindaceae & 1 & 0.2 & 0.7 & 0.9 \\
\hline Inga marginata & Leguminosae & 2 & 0.23 & 1.3 & 1.5 \\
\hline Eugenia florida & Myrtaceae & 1 & 0.2 & 0.7 & 0.9 \\
\hline Beilschmiedia emarginata & Lauraceae & 2 & 0.16 & 1.3 & 1.5 \\
\hline Guarea macrophylla & Meliaceae & 2 & 0.15 & 1.3 & 1.5 \\
\hline Machaerium stipitatum & Leguminosae & 1 & 0.14 & 0.7 & 0.8 \\
\hline Pterocarpus cf. rohrii & Leguminosae & 2 & 0.13 & 1.3 & 1.4 \\
\hline Eugenia riedeliana & Myrtaceae & 1 & 0.12 & 0.7 & 0.8 \\
\hline Byrsonima ligustrifolia & Malpighiaceae & 2 & 0.12 & 1.3 & 1.4 \\
\hline Alchornea triplinervia & Euphorbiaceae & 1 & 0.12 & 0.7 & 0.8 \\
\hline Sp. 3 & & 1 & 0.11 & 0.7 & 0.8 \\
\hline Sp. 1 & & 1 & 0.09 & 0.7 & 0.7 \\
\hline Monimiaceae sp.1 & Monimiaceae & 1 & 0.07 & 0.7 & 0.7 \\
\hline Mollinedia clavigera & Monimiaceae & 1 & 0.07 & 0.7 & 0.7 \\
\hline Lonchocarpus muehlbergianus & Leguminosae & 1 & 0.07 & 0.7 & 0.7 \\
\hline Swartzia aff. acutifolia & Leguminosae & 1 & 0.07 & 0.7 & 0.7 \\
\hline Calyptranthes concinna & Myrtaceae & 1 & 0.07 & 0.7 & 0.7 \\
\hline Alseis floribunda & Rubiaceae & 1 & 0.07 & 0.7 & 0.7 \\
\hline Myrtaceae sp.1 & Myrtaceae & 1 & 0.06 & 0.7 & 0.7 \\
\hline Rudgea cf. jasminoides & Rubiaceae & 1 & 0.05 & 0.7 & 0.7 \\
\hline Lauraceae sp.1 & Lauraceae & 1 & 0.05 & 0.7 & 0.7 \\
\hline
\end{tabular}

area. This methodology proved to be a useful tool in the selection of forested areas to exemplify the succession dynamics or chronosequence that occurs in the study site. Our analysis was favoured by the occurrence of two sets of photos in 1973, where in an interval of two months we identified an area of cut forest, which corresponds in December 1997 to a forest fragment with 24.5 years old.

The phytosociological survey carried out in the chronosequence was considered sufficient to achieve the main objective of this work, i.e., the characterization of the main species involved in the secondary succession process at the study site. The transect with 0.1 ha used to assess the successional stages produced a relatively fast and accurate result, as suggested by Shugart (1984), who indicates a surface area around 0.1 ha adequate for application of "gap models". However, to assess the tree species diversity (species richness, abundance and Shannon diversity) it would be recommended a larger sampling area as suggested by He et al. (1996).

The species composition along the forest succession phases at the study site indicates a different pattern of forest succession, where Piptadenia gonoacantha (Mimosaceae) substitutes the species generally found at the initial succession of Atlantic Forest over acidic soils, Tibouchina spp. (Melastomataceae) (Loefgren 1898, Leitão Filho et 
al. 1993). The occurrence of this kind of soil represents a typical situation in the Atlantic Forest, where most soils are usually found to be acidic, highly leached and nutrient deficient, although there is great diversity of specific soil types within the humid tropics (Jordan 1985).

The results indicate that $P$. gonoacantha only occurs over the calcareous soil, while T. pulchra showed a marked decrease in importance when compared with the successional dynamics outside the limestone area, e.g. in an area close to the study site (Torezan 1995, Godoy 2001). This suggests a competitive exclusion, where $P$. gonoacantha a superior competitor in this kind of environment, displaces the species T. pulchra.

The occurrence of angiosperm families showed a clear dominance of Leguminosae throughout succession and a decrease of importance of Melastomataceae in the more mature forest. In the early succession, Symplocaceae and Flacourtiaceae were important because the high number of individuals of a single species Symplocos laxiflora and Casearia sylvestris, respectively. In the intermediary stage, Myrsinaceae was important because of the number of individuals of Myrsine coriacea, an understorey species, showing clearly the beginning of stratum development. The importance of Leguminosae is due to the highest basal area shown by the relative dominance. The most important species was $P$. gonoacantha that accounted for more than $30 \%$ of total basal area of the community and more than $90 \%$ of the basal area from leguminous species.

In the older stage, Leguminosae was again the most important family, accounting for almost $30 \%$ of total basal area and showing the occurrence of nine species. Lauraceae and Myrtaceae were also important because the high number of individuals and species.

Considering the inventory as a whole, Leguminosae was the most important family followed by Myrsinaceae, Myrtaceae, Annonaceae, Lauraceae, Rubiaceae and Melastomataceae, which is, except for the dominance of Leguminosae, a similar result as found by Leitão Filho et al. (1993) for secondary forest in areas protected from air pollution in Cubatão, São Paulo. In addition, these families are considered by Oliveira Filho \& Fontes (2000), except Myrsinaceae, as having the highest number of species on the flora of areas of Ombrophylous Dense Forest.
Despite of the fact that calcareous soils cover more than 30\% of earth's surface (Marschner 1995), their occurrence is less than $7 \%$ in the Brazilian territory (Karmann 1994). In addition, the main areas of karst landscape in Brazil are situated in dry and/or seasonal zones, where dominant vegetation are Cerrado and Deciduous Seasonal Forest. Whitmore (1984) indicates that karst landscape is absent of the humid tropics of Africa and it is rare in Latin America, except for Caribbean region. The author also describes karst occurrence in a small part of the tropical Far East (China, Indo-Chine, Sumatra, Malaya, Sarawak, Java and New Guinea), where the forests over limestone have several characteristic species occurring in habitats normally drier than the surrounding areas, stressing that acidic soils are common because peat occurrence.

The Atlantic Forest over calcareous soil found at the study site is important for Neotropical Forest Biodiversity because it is probably the only site where it occurs in Brazil, being a very important genetic bank of threatened species such as Ocotea catharinensis Mez - Lauraceae (SBB 1992); Aspidosperma ramiflorum Müll. Arg. - Apocynaceae; Myrocarpus frondosus Allemao - Fabaceae; Chrysophyllum inornatum Mart. - Sapotaceae; and Euterpe edulis Mart. - Arecaceae (www.bdt.org.br/ redflora/).

These results provided a background to study the forest architectural growth, successional dynamics and species regeneration strategies, and for the study of ecophysiology of nitrogen use strategies by tree species and the relations with successional status (Aidar 2000).

The characterization of this locally restricted Atlantic Forest successional pattern is decisive in supporting actions taken to rehabilitate degraded lands, and can be a potential tool for sustainable tropical forest management, including the potential to serve as carbon offset mechanism (Silver et al. 2000), both inside and around the Alto Ribeira Touristic State Park boundaries.

Acknowledgements - We thank Prof. Dr. Ricardo R. Rodrigues ESALQ-USP for the interest and help in the identification of the plants; Prof. Dr. Jean Paul Metzger IB-USP for the cooperation in the development of this work; Prof. Dr. Marcos $\mathrm{S}$. Buckeridge for the english revision and the continuous encouraging support; our field workers, José da Mota, Eufrásio da Mota e Orlei Lopes, that made our sampling possible; the people from PETAR-Instituto Florestal for their permission 
to work in the Conservation Unit, Roberto Burgui in memoriam; and Silvia Nogueira for help in the aerial photographic documentation.

\section{References}

AIDAR, M.P.M. 2000. Ecofisiologia das estratégias de utilização de nitrogênio em árvores da floresta neotropical. Tese de doutorado, Universidade Estadual de Campinas, Campinas.

BROWN, S.; LUGO, A.E. 1990. Tropical secondary forests. Journal of Tropical Ecology 6:1-32.

BRIEGER, F.G. 1969. Patterns of evolutionary and geographical distribution in Neotropical orchids. Biological Journal of the Linnean Society 1:197-217

BROKAW, N.V.L. 1985. Gap-phase regeneration in tropical forest. Ecology 66:682-687

CAMARGO, O.A., MONIZ, A.C., JORGE, J.A. \& VALADARES, J.M.A.S. 1986. Métodos de análise química, mineralógica e física de solos do Instituto Agronômico de Campinas. Boletim Técnico 106. Instituto Agronômico de Campinas, Campinas.

CHAZDON R. 1998. Tropical forests (log 'em or leave 'em). Science 281:1295-1296.

EMRICH, A., POKORNY, B. \& SEPP, C. 2000. The significance of secondary forest management for development policy. Series N. FTWF-18e, GTZ, Eschborn.

FAO 1996. Forest resources assessment 1990. Survey of tropical forestcover and study of change processes. Forestry Paper 130, FAO. Rome.

FAO-ISRIC-ISSS. 1998. World reference base for soil resources. World Soil Resources Reports 84. FAO, ISSS and ISRIC, Rome.

GODOY, J.R.L. 2001. Estrutura e composição específica da Mata Atlântica secundária de encosta sobre calcário e filito, no Parque Estadual Turístico do Alto Ribeira, Iporanga, SP. Dissertação de mestrado, Universidade de São Paulo, São Paulo.

GÓMEZ-POMPA, A. \& BURLEY, F.W. 1991. The management of natural tropical forests. In Rain forest regeneration and management. (A. Gómez-Pompa, T.C. Whitmore \& M. Hadley, eds.) Man and Biosphere, series vol. 6, UNESCO/Parthenon Publ., Paris, p.3-18.

GUTJAHR, M.R. 1993. Critérios relacionados à compartimentação climática de Bacias Hidrográficas: A Bacia do Rio Ribeira de Iguape. Dissertação de mestrado, Universidade de São Paulo, São Paulo.

HE, F.L., LEGENDRE, P. \& LAFRANKIE, J.V. 1996. Spatial pattern of diversity in a tropical rain forest in Malaysia. Journal of Biogeography 23:57-74

IBGE. 1992. Manual técnico da vegetação brasileira. Manuais técnicos em geociências, v.1, Instituto Brasileiro de Geografia e Estatística, Rio de Janeiro.

JOLY, C.A., AIDAR, M.P.M., KLINK, C.A., MCGRATH, D.G., MOREIRA, A.G., MOUTINHO, P., NEPSTAD, D.C., OLIVEIRA, A.A., POTT, A., RODAL, M.J.N. \& SAMPAIO, E.V.S.B. 1999. Evolution of the Brazilian phytogeography classification systems: implications for biodiversity conservation. Ciência e Cultura 51:331-348.

JORDAN, C.F. 1985. Nutrient cycling in tropical forest ecosystems. John Wiley and Sons, New York.
KARMANN, I. 1994. Evolução e dinâmica atual do sistema cárstico do alto Vale do rio Ribeira de Iguape, sudeste do estado de São Paulo. Tese de doutorado, Universidade de São Paulo, São Paulo.

LEITÃO FILHO, H.F., PAGANO, S.N., CESAR, O., TIMONI, J.L. \& RUEDA, J.J. 1993. Ecologia da Mata Atlântica em Cubatão. Ed. UNESP/EDUSP, São Paulo.

LOEFGREN, A. 1898. Ensaio para uma distribuição dos vegetaes nos diversos grupos florísticos no estado de São Paulo. Reditado em: A vegetação do Estado de São Paulo, 1970. (G. Eiten, ed.). Boletim da Comissão Geológica e Geográfica de São Paulo, vol. 7. São Paulo.

MARSCHNER, H. 1995. Mineral nutrition of higher plants. Academic Press, London.

MORI, S.A., BOOM, B.M. \& PRANCE, G.T. 1981. Distribution patterns and conservation of Eastern Brazilian coastal forest tree species. Brittonia 33:233245.

MÜLLER-DOMBOIS, D. \& ELLENBERG, H. 1974. Aims and methods in vegetation ecology. Willey and Sons, New York

MYERS, N., MITTERMEIER, R.A., MITTERMEIER, C.G., DA FONSECA, G.A.B. \& KENT, J. 2000. Biodiversity hotspots for conservation priorities. Nature 403:853858.

OLIVEIRA FILHO, A. \& FONTES, M.A. 2000. Patterns of floristic differentiation among Atlantic Forests in southeastern Brazil, and the influence of climate. Biotropica 32:793-810.

SBB 1992. Centuria plantarum brasiliensium extintions minitata. $1^{\text {a }}$ ed. Sociedade Botânica do Brasil, Rio de Janeiro.

SHUGART, H.H. 1984. A theory of forest dynamics: the ecological implications of forest succession models. Springer Verlag, Berlin.

SILVER, W.L., OSTERTAG, R. \& LUGO, A.E. 2000. The potential for carbon sequestration through reforestation of abandoned tropical agricultural and pasture lands. Restoration Ecology 8:394-407.

SOS MATA ATLANTICA 1998. Evolução dos remanescentes florestais e ecossistemas associados do domínio Mata Atlântica no período 1990-1995. Fundação SOS Mata Atlântica/INPE, São Paulo.

SUBLER, S. \& UHL, C. 1990. Japanese agroforestry in Amazonia: a case study in Tomé-açu, Brazil. In: Alternatives to deforestation. Steps towards sustainable use of amazon rain forest. (A.B. Anderson, ed.). Columbia University Press, New York. p.152-166

TABARELLI, M., VILLANI, J.P., MANTOVANI, W. 1993. Aspectos da sucessão secundária em trecho de floresta atlântica no Parque Estadual da Serra do Mar, SP. Revista do Instituto Florestal 5:99-112.

TABARELLI, M., VILLANI, J.P. \& MANTOVANI, W. 1994. Estudo comparativo da vegetação de dois trechos de floresta secundária no Núcleo Santa Virgínia, Parque Estadual da Serra do Mar, S.P. Revista do Instituto Florestal 6:1-11.

TABARELLI, M., MANTOVANI, W. 1999. A regeneração de uma floresta tropical montana após corte e queima (São Paulo - Brasil). Revista Brasileira de Biologia 59:239-250. 
TOREZAN, J.M.D. 1995. Estudo da sucessão secundária, na floresta ombrófila densa submontana, em áreas anteriormente cultivadas pelo sistema de "coivara", em Iporanga - S.P. Dissertação de mestrado, Universidade Federal do Paraná, Curitiba.

TORRES, R.B., MARTINS, F.R. \& GOUVEA, L.S.K. 1997. Climate, soil and tree flora relationships in forests in the State of São Paulo, southeastern Brazil. Revista Brasileira de Botânica 20:41-49.
UHL, C., NEPSTAD, D., BUSCHBACHER, R., CLARK, K., KAUFMAN, B. \& SUBLER, S. 1990. Studies of ecosystem response to natural and anthropogenic disturbances provide guidelines for designing sustainable land-use system in Amazonia. In Alternatives to deforestation. Steps towards sustainable use of amazon rain forest. (A.B. Anderson, ed.). Columbia University Press, New York, p.24-42.

VELOSO, H.P. 1982. Fitogeografia brasileira. Projeto Radambrasil. Bol. Técnico Ser. Vegetação 1. Salvador.

WHITMORE, T.C. 1984. Tropical rain forests of the far west. Clarendon Press $2^{\text {nd }}$ ed. Oxford. 